\title{
Classificação Internacional de Atenção Primária: capturando e ordenando a informação clínica
}

\author{
The International Classification of Primary Care: \\ capturing and sorting clinical information
}

Gustavo Gusso (https://orcid.org/0000-0002-7488-7535) ${ }^{1}$

${ }^{1}$ Faculdade de Medicina, Universidade de São Paulo. Av. Enéas Carvalho de Aguiar 155, Pinheiros. 05403-000 São Paulo SP Brasil.gustavo.gusso@usp.br

\begin{abstract}
The International Classification of Primary Care-2 (ICPC-2) is the result of forty years of continuous development. It originates in the second half of the twentieth century after the concern of general practitioners about the need to record and encode data specifically related to primary care, both in the reasons for encounter and procedures and conditions or diagnoses. The World Health Organization endorsed the classification, as did the developer committee after the Alma-Ata meeting, since it also identified specific needs. Two forms of use are employed now in gathering information: by encounter or by an episode of care. The latter is more complex and controversial. Recently, an eleventh version of the International Classification of Diseases has been released, and the third edition of ICPC is being developed. One cannot predict how new technologies, classifications, and international organizations will interact. The role of front line health professionals and patients will define the course.

Key words International classification of primary care, General practice, Primary health care
\end{abstract}

Resumo A Classificação Internacional de Atenção Primária-2 (CIAP-2) é fruto de quarenta anos de desenvolvimento contínuo. Tem origem na segunda metade do século XX, a partir da inquietação de médicos gerais com a necessidade de se registrar e codificar dados especificamente relacionados à atenção primária, tanto nos motivos de consulta quanto nos procedimentos e nas condições ou diagnósticos. A Organização Mundial de Saúde chancelou a classificação bem como o seu comitê desenvolvedor após o encontro de Alma-Ata, pois também identificou necessidades especificas. Hoje há essencialmente duas formas de uso na coleta de informações: por encontro ou por episódio de cuidado. A segunda forma é mais complexa e controversa. Recentemente foi lançada a décima primeira versão da Classificação Internacional de Doenças, enquanto que a CIAP-3 esta sendo desenvolvida. Não há como prever como vão interagir com as novas tecnologias, as classificações e os organismos internacionais. O protagonismo dos profissionais da ponta e dos pacientes tem potencial de definir a direção.

Palavras-chave Classificação internacional de atenção primária, Clinica geral, Atenção primária à saúde 


\section{História}

Desde a Lista Internacional de Causas de Morte de 1893, até sua sexta revisão em 1948, que incluiu morbidade em uma lista única dando origem à Classificação Internacional de Doenças (CID), pesquisadores têm uma ferramenta para classificar e estudar a morte e a doença. Na metade do século XX, clínicos gerais de diversos países, em especial da Inglaterra, passaram a pesquisar as demandas dos pacientes das suas próprias clinicas $^{1,2}$.

Nesta época, um médico de família inglês publicou um artigo que demonstrou a partir da sua prática algumas especificidades importantes da atenção primária à saúde ${ }^{3}$. Segundo Donald Crombie, o estabelecimento de um diagnóstico preciso foi possível em menos de $50 \%$ das consultas e isto ocorreu principalmente nas situações em que apenas a história clínica e o exame físico focado foram realizados. Exames complementares em geral não ajudaram no estabelecimento do diagnóstico, mas a opinião de um especialista colaborou. As cinco conclusões elencadas por Crombie continuam atuais, corroboradas pelo progresso da medicina ${ }^{3}$ :

muitas "condições" são autolimitadas e requerem apenas observação ativa;

pacientes frequentemente demandam apenas serviços administrativos;

a fisiopatologia de base de "condições" indefinidas e não graves é desconhecida;

os exames laboratoriais e a tecnologia não colaboram para o complexo processo diagnóstico de "condições" freqüentes;

é má prática tentar vigorosamente a definição de um diagnóstico para uma “condição" vaga especialmente se esta está acompanhada de componentes psicológicos.

Crombie utiliza o termo "minor disabilities", traduzido como "condições", o qual, por ser uma aproximação, foi colocado entre aspas. Já “condições”, com aspas, foi o escolhido neste texto para designar a descrição ou o diagnóstico médico, enquanto que "diagnóstico" foi evitado por nem sempre se tratar de doença. O termo que mais vinha sendo utilizado era "problemas", mas "condição” tem sido substituído por ser mais amplo. Por exemplo, gravidez é uma “condição”, mas não um "problema"

O último item merece destaque, embora ainda não se tenha totalmente a dimensão dos efeitos nocivos que o exagero na busca de um diagnóstico etiológico possa acarretar. Recomenda-se vigorosamente não tentar definir um diagnóstico para uma “condição" vaga, mas buscar a acurácia, pois um eventual erro pode levar a sérios prejuízos. A primeira classificação para uso na clínica geral foi desenvolvida em 1959 pelo Colégio Britânico de Clínicos Gerais ${ }^{4}$. Médicos de família e clínicos gerais de muitos países notaram a importância da taxonomia neste campo específico, de modo a evitar o uso inadequado de uma classificação baseada apenas nas doenças e nas causas de morte. Isto poderia ser perigoso, uma vez que, escolhida uma doença como diagnóstico, o tratamento estaria autorizado; porém, como já havia sido demonstrado, um diagnóstico específico só seria possível em aproximadamente $50 \%$ dos encontros do médico com o paciente.

A Classificação por Motivo de Visita (Reason for Visiting Classification ou RVC) e o seu processo de desenvolvimento nos Estados Unidos da América, incluindo a criação da Pesquisa Nacional sobre Atenção Médica Ambulatorial (National Ambulatory Medical Care Survey ou NAMCS), pelo Centro Nacional para Estatísticas da Saúde (National Centre for Health Statistics ou NCHS), em 1973, foi um passo importante ${ }^{5,6}$. O primeiro sistema de classificação de motivos da consulta foi desenvolvido por Sue Meads, uma taxonomista, filha de um médico rural, para uso na NAMCS ${ }^{7}$.

Um segundo passo foi a organização dos generalistas. Médicos de família e clínicos gerais, interessados no campo da taxonomia, que se encontraram em 1972 durante a Conferência Mundial dos Clínicos Gerais/Médicos de Família, a qual fundou a WONCA (Organização Mundial dos Médicos de Família ou World Organization of National Colleges, Academies and Academic Associations of General Practice/ Family Medicine), em Melbourne, Austrália, concluíram que deveriam desenvolver um sistema de classificação próprio . Os clínicos gerais Bent Bentsen (Noruega), Charles Bridges-Webb (Austrália), Robert Westbury (Canadá), Philip Sive (Israel) estavam trabalhando neste tema ${ }^{8-11}$. Eles organizaram, então, o Comitê de Classificação da WONCA (cujo nome seria modificado para Comitê Internacional de Classificação da WONCA ou WONCA International Classification Committee - WICC) para desenvolver um sistema baseado na CID 8. Este grupo organizou uma lista de "condições" comumente encontradas na atenção primária baseada na CID 8 e testou-a em 300 consultórios de nove países resultando na Classificação Internacional de Problemas de Saúde em Atenção Primária (International Classification of Health Problemas in Primary Care ou ICHPPC), apresentada em 1974 e publicada em 1975 pela Associação Hospita- 
lar Americana e pelo Colégio Real Britânico de Clínicos Gerais. A mesma foi revisada, enquanto que a segunda versão (ICHPPC-2) foi publicada em 1979 para manter a comparabilidade com a CID 9 (Figura 1). Apesar de elaborada por médicos de família para uso na prática diária, apenas o último capitulo era dedicado aos sinais e sintomas, refletindo a perspectiva dos pacientes. As duas versões da ICHPPC eram, neste sentido, mais um recorte da CID 8 e 9 do que uma classificação voltada para a atenção primária.

Concomitantemente ao desenvolvimento da ICHPPC, existia desde 1977 um direcionamento do Grupo Norte Americano de Pesquisa em Atenção Primária (North American Primary Care Research Group ou NAPCRG) para o desenvolvimento da Codificação do Processo de Cuidado em Atenção Primária (Process Code for Primary Care $)^{12}$ envolvendo diagnóstico, prevenção, exame, tratamento e demais procedimentos terapêuticos. A primeira versão era um produto do NAPCRG que formalizou seu próprio comitê de classificações. Este se juntou ao Comitê de Classificação da WONCA e desenvolveu uma versão internacional chamada de Classificação Internacional de Processos de Cuidado em Atenção Primária (International Classification of Process in Primary Care ou IC-Process-PC), a qual foi testada em dez países, envolvendo aproximadamente 100 médicos, sendo finalmente publicada em 1986. Foram incluídas condutas típicas da atenção primária, como "observação", "observação ativa" (watchful waiting), "não intervenção" ou "repouso como modalidade terapêutica".

Depois da conferência de Alma-Ata em 1978, a Organização Mundial da Saúde identificou a importância da informação e de ferramentas apropriadas para a atenção primária à saúde e nomeou uma força tarefa para desenvolver a Classificação por Motivos da Consulta (CMC ou, em inglês, Reason for Encounter Classification $R F E C)$ que iria focar na perspectiva do paciente e não na doença ${ }^{13-15}$. O motivo da Consulta (MC) é definido como "um termo acordado que transmite as razões pelas quais um paciente entra no sistema de saúde e representa as demandas desta pessoa"13. A maioria dos membros deste grupo de trabalho da Organização Mundial da Saúde era do Comitê de Classificação da WONCA. A Classificação por Motivos da Consulta foi testada em 1980 na Holanda ${ }^{14}$ e em nove países, em 1983 (Brasil, Austrália, Barbados, Hungria, Malásia, Holanda, Noruega, Filipinas e Estados Unidos da América $)^{16}$. No Brasil, o estudo ficou sob a responsabilidade do Centro Brasileiro de Classificação de Doenças, coordenado pelo Professor Ruy Laurenti. A parte brasileira do piloto foi conduzida pelos pesquisadores Ruy Laurenti e Maria Lucia Lebrão e, além de médicos, utilizou enfermeiros e agentes comunitários de saúde. O estudo foi apoiado pela OMS, WONCA e NCHS ${ }^{14,17}$.

$\mathrm{O}$ processo de desenvolvimento da Classificação por Motivos da Consulta (CMC) permitiu concluir que as três principais classificações até então desenvolvidas por pesquisadores vinculados à atenção primária ou a médicos de família e publicadas (Classificação Internacional de Problemas de Saúde em Atenção Primária - ICHPPC, Classificação por Motivos de Visita - RVC e Classificação Internacional de Processos de Cuidado em Atenção Primária - IC-Process-PC) seriam contempladas na CMC, porque esta previa também os componentes para procedimentos e diagnósticos.

O resultado do trabalho do grupo constituído por membros da OMS e da WONCA foi

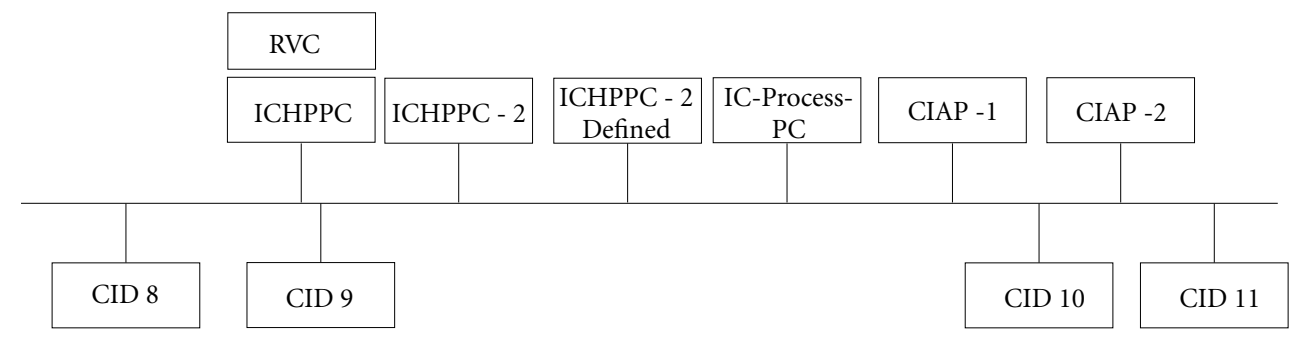

Figura 1. Linha do tempo - Histórico do desenvolvimento da Classificação Internacional de Doenças (linha inferior) e Classificação Internacional de Atenção Primária (linha superior). 
uma classificação biaxial com dezesseis capítulos baseados no critério anatômico (e no sistema orgânico), além dos sete componentes que compõem cada capítulo. Depois do primeiro piloto, na Holanda, foi incluído o capítulo de reprodução ("Gravidez, Parto e Planejamento Familiar") ${ }^{14}$. Utilizar a anatomia e o sistema orgânico como critério básico e incluir os capítulos "Geral e Inespecífico", "Psicológico" e "Problemas Sociais" para desenvolver o sistema de classificação foi uma decisão importante, porque estes capítulos refletiriam prioritariamente os aspectos do paciente e não as doenças, as manifestações ou as etiologias. Subdivisões por etiologias, como trauma, doenças infecciosas, tumor e anomalias congênitas, foram incluídas no sétimo componente (diagnósticos e doenças) e distribuídos nos respectivos capítulos (Quadro 1). Isto foi de fundamental importância porque evitou possíveis conflitos com os princípios classificatórios como a mutualidade excludente ${ }^{18}$, ou seja, cada conceito ser encontrado apenas em um local. Se fossem criados capítulos específicos para doenças infecciosas ou traumas, não haveria uma hierarquia clara entre localização e etiologia e estaria permitida a dupla codificação, uma vez que a doença poderia estar nos capítulos etiológico e anatômico. Isto ocorre com a CID, que, desde a nona revisão, utiliza o sistema "cruz-asterisco" para justamente tentar evitar a dupla codificação ${ }^{19}$.

$O$ principal critério para uma rubrica ser incorporada passou a ser a regra arbitrária de prevalência de 1:1000 na população atendida ${ }^{13}$. O grupo de trabalho entendeu que seria a prevalência mínima para um generalista se responsabilizar pela condição. Quando a condição era menos prevalente, ficava em uma rubrica chamada de "rag bag" (na tradução literal, "cesta de trapos") que passaram a ser, em cada capítulo, os códigos 29 para sintomas e 99 para diagnósticos.

Apesar de aparentemente simples, a CMC foi inspirada em classificações que estavam em uso ou que vinham sendo desenvolvidas na época ${ }^{13}$. O primeiro componente (queixas e sintomas) usou elementos da Classificação por Motivos de Visita (RVC) do braço ambulatorial da Pesquisa Nacional sobre Atenção Médica Ambulatorial (NAM$\mathrm{CS})^{5,20-23}$; do segundo ao sexto componentes (processos de cuidado) foram baseados na 9a Revisão da Classificação Internacional de Procedimentos em Medicina ${ }^{24}$ e na nova Codificação de Processo de Cuidado em Atenção Primária do NAP$\mathrm{CRG}^{25,26}$; o sétimo componente (diagnósticos e doenças) foi elaborado a partir da ICHPPC-2- defined. Os capítulos P (psicológico) e Z (social) foram inspirados na classificação triaxial da $\mathrm{OMS}^{27}$, baseada nos eixos psicológico, social e orgânico que nunca foi colocada em prática. Desta maneira, a CMC englobou os Motivos da Consulta, os Processos de Cuidado e as Doenças.

Embora houvesse sinais de que a CMC pudesse ser o núcleo da $10^{\mathrm{a}}$ revisão ${ }^{28}$, pois havia incorporado doenças e processos além dos motivos da consulta, a OMS mudou esta direção e decidiu não alterar a estrutura tradicional da CID. Mas a WONCA foi adiante e publicou a CMC após

Quadro 1. Estrutura biaxial da CIAP.

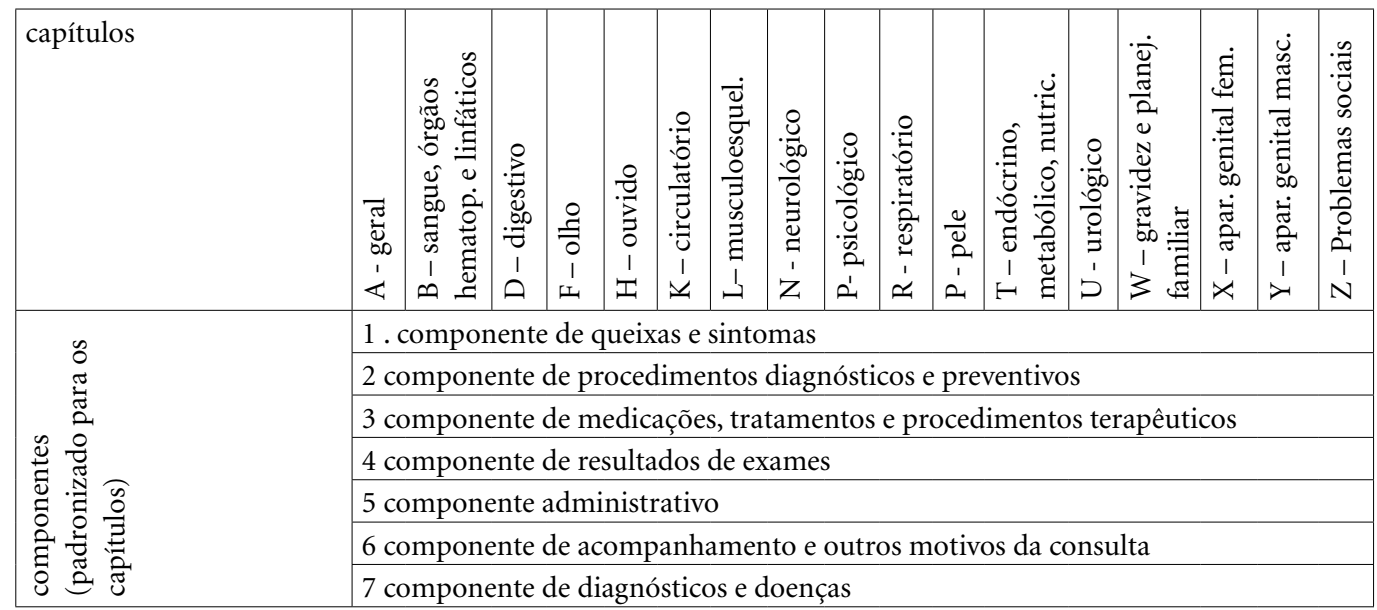


os pilotos como a primeira versão da Classificação Internacional de Atenção Primária (CIAP ou International Classification of Primary Care ou ICPC da sigla em inglês) em $1987^{29}$, com possibilidade de mapeamento com a CID 9 (Figura 1). A CIAP passou a fazer parte da família de classificações da OMS. Uma revisão foi publicada em $1993^{30}$. A segunda versão da CIAP, com critérios de inclusão e exclusão, foi lançada em 1998, e desde então traduzida para diversos idiomas. Há uma versão sumária que pode ser impressa em uma folha de papel A4, também disponível no site da Sociedade Brasileira de Medicina de Família e Comunidade ${ }^{31}$.

A CID foi programada para ser revisada a cada dez anos. Houve uma regularidade no início, mas entre a nona e a décima revisão ocorreu um atraso que se acentuou entre a décima e a décima primeira. $\mathrm{O}$ avanço da informatização e a criação do SNOMED International, a partir da união da nomenclatura inglesa conhecida como "Read Codes" e o Systematized Nomenclature of Medicine (SNOMED) do Colégio Americano de Patologistas, trouxe uma complexidade para o universo das classificações, embora o SNOMED seja uma nomenclatura ${ }^{32}$. Nomenclaturas são comumente descritas como sinônimos de terminologias e, ao contrário das classificações, não precisam seguir regras como possuir mutualidade excludente, ter uma hierarquia bem definida e ser completa no campo no qual pretende atuar ${ }^{18}$. A Organização Mundial da Saúde e as suas comissões, que desenvolvem a CID, demoraram a encontrar um caminho compatível com o rápido avanço da informatização na saúde ${ }^{33}$.

\section{Regras essenciais}

A CIAP-2 pode ser usada tanto para a codificação dos registros de motivos de consulta, que é a síntese do subjetivo na sistematização do Registro Clinico Orientado por Problemas ${ }^{34}$, quanto de processos de cuidado (dados objetivos ou plano) e condições (avaliação).

Regras para codificação do Motivo de Consulta (síntese do campo Subjetivo do SOAP) ${ }^{13}$ :

$O$ paciente tem que concordar com o código selecionado, portanto, os critérios de inclusão e exclusão da rubrica não valem para o registro do Motivo da Consulta e sim o termo usado pelo paciente.

A palavra é mais importante que o conceito (codificar "icterícia" caso o paciente use este termo, mesmo que ele não saiba exatamente do que se trata).
Escolher o capítulo mais específico de acordo com a maneira como o paciente se expressa. Por exemplo, se o paciente relatar que tem dor torácica, deve ser questionado se este acha que é relacionado ao coração (capitulo K), ao pulmão (capítulo R), muscular (capítulo L) ou se não sabe (capítulo A)

Pode-se usar os três eixos da CIAP-2: sintomas (códigos 1 a 29), processo de cuidado (códigos 30 ao 69), ou diagnósticos (códigos 70 ao 99).

Regras para o registro das "Condições" (síntese do campo Avaliação do SOAP) ${ }^{13}$ :

Deve-se buscar o máximo de especificidade: se houver dados suficientes para um diagnóstico, mesmo sem a existência de exame complementar, este deve ser registrado e codificado; caso não haja dados suficientes, deve-se buscar o mais alto grau de especificidade, o que frequentemente implica no uso do "sintoma como diagnóstico"35 e a replicação do(s) código(s) do Motivo da Consulta.

Os critérios de inclusão ou exclusão não podem ser usados para formular diagnóstico, mas apenas para consulta após o diagnóstico formulado, ou seja, a classificação não deve ser usada como protocolo.

Colocar dois códigos/conceitos quando necessário. Por exemplo, se houver fibrilação atrial e ansiedade e não houver evidências que um causa o outro, registrar como duas condições distintas.

Evitar capítulo Geral (A) quando possível e códigos/conceitos de Gestantes (capítulo W) quando a "condição" não tiver relação com gestação

Neste campo, o paciente não precisa necessariamente concordar com a "condição" registrada, ao contrário do campo Motivo de Consulta, exceto se for alguma condição social (capítulo Z). Esta regra protege o médico de uma pratica moralista.

Só se deve usar os códigos 1 a 29 ou 70 a 99; enquanto que os códigos de processo (30 a 69) de cuidado não devem ser usados para codificar "condições".

Os componentes $\mathrm{O}$ e $\mathrm{P}$ de processo de cuidado devem usar apenas os códigos 30 a 69, os quais se repetem a cada capítulo.

\section{Formas de uso}

Há essencialmente duas formas de se usar a Classificação Internacional de Atenção Primária, tanto no papel quanto em meio digital: por encontro ou por episódio de cuidado. Quando se usa por encontro, cada consulta é codificada 
sem levar em conta a continuidade do cuidado de cada "condição". Por exemplo, codifica-se fraqueza em um consulta e anemia na próxima, mas não se relaciona fraqueza a anemia como evolução do cuidado, neste caso, especificado ao longo do tempo através de exame laboratorial. Cada consulta ou encontro é codificado de forma independente do anterior. Quando se codifica por encontro, mesmo que um paciente seja atendido na mesma semana pelo mesmo quadro de gripe, a condição é contabilizada duas vezes. Portanto, há um impacto na incidência das condições. Por outro lado, caso os motivos da consulta sejam registrados relacionados às suas respectivas condições, criando-se "subencontros", é possível uma analise preditora. Ou seja, é possível responder às perguntas: "dado tais motivos de consulta, quais foram as condições mais registradas?" e "dadas as condições mais registradas, quais foram os motivos das consultas apresentados?". Portanto, embora não seja mandatória a criação de "subencontros" com um registro SOAP para cada condição, esta técnica potencializa a analise $\mathrm{e}^{36,37}$.

Episódio de cuidado é definido como "todo tipo de atenção prestada a um determinado indivíduo que apresente uma condição, um problema de saúde ou uma doença"13. Portanto, o conceito é diferente de episódio de sintoma ou episódio da doença, pois é baseado na atenção prestada. Os episódios (espaço de tempo) podem ter um ou mais encontros. A maior parte dos episódios de cuidado tem apenas um encontro ${ }^{35}$.

No episódio ilustrado na Figura 2, o rótulo da "condição" seria fraqueza ao final do primeiro encontro, passaria para anemia inespecífica ao final do segundo e então a câncer de cólon ao final do terceiro. Este tipo de registro colabora na resposta de perguntas epidemiológicas como "qual a probabilidade de uma pessoa com fraqueza ter câncer de cólon?"

O registro por episódio de cuidado é mais complexo e também contestado, pois implica em necessariamente criar "subencontros" para que cada "condição" seja registrada de forma independente e, assim, garantir que está se dando continuidade a cada episódio. As críticas se concentram especialmente na impossibilidade de trabalhar cada condição de forma tão independente quanto este tipo de registro requer.

\section{CIAP-3}

A terceira versão da Classificação Internacional de Atenção Primária começou a ser desen-
Antes da primeira consulta

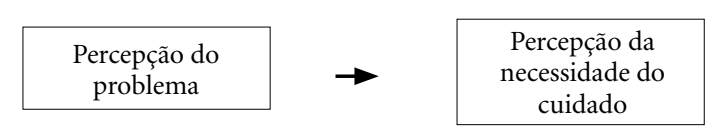

cuidado

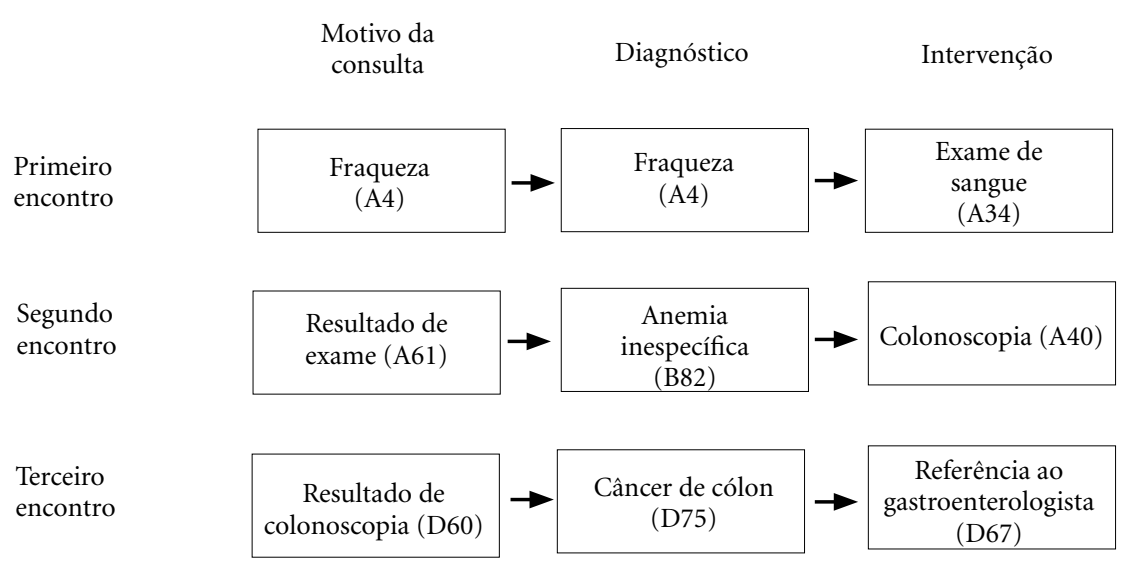

Figura 2. Exemplo de Episódio de Cuidado com os códigos da CIAP $2^{13}$. 
volvida pelo Wonca International Classification Committe (WICC). No início, as discussões estavam focadas na estrutura, já que a segunda versão, por ter três dígitos, sendo uma letra e dois números, não permitia uma grande extensão. Portanto, foi decidido que a terceira versão teria quatro dígitos como a CID 11, porém sempre duas letras no início e dois números na sequência.

Em 2015 iniciou-se a organização de um consórcio para financiar o desenvolvimento desta terceira versão, já que os membros do WICC eram voluntários. Aderiram à iniciativa: Wonca Europa, Bélgica, Brasil, Finlândia, França e Holanda e o grupo de trabalho formado por membros indicados pelos participantes do consórcio, o qual iniciou as atividades em janeiro de 2018 com previsão de concluir em três anos ${ }^{38}$. Os primeiros relatórios do consórcio apresentados nas reuniões anuais da WICC de 2018 e 2019 e a primeira versão disponibilizada para os membros do consórcio apontam para as seguintes características:

As rubricas terão quatro dígitos sendo duas letras seguidas por dois números;

Na maioria dos capítulos a primeira letra será a mesma da CIAP 2, que segue a regra mnemônica do órgão segundo o termo em inglês (B de "blood", S de "skin", P de "psychologic");

A segunda letra será, na maioria dos capítulos: S para sintoma e D para diagnóstico. Mas, ao contrário da segunda versão, ao invés de cada parte ter apenas 30 possibilidades de rubricas, $\mathrm{k}$ passa a ter 99 (de 01 a 99 );

Os capítulos relacionados aos aparelhos genitais masculino e feminino serão fundidos, dando origem ao capítulo G (Gênero);

Haverá um capítulo, ou subcapítulo, para "motivos de contatos com serviços de saúde", como rastreamento, vacinação, contracepção e estilo de vida;

Será criado um capítulo para rubricas relacionadas à funcionalidade, como, por exemplo, capacidade de dirigir, de se vestir, de se relacionar, de se manter aberto a experiências; o qual poderá ser mapeado para a Classificação Internacional de Funcionalidade ${ }^{39}$;

Haverá extensões para qualificar as rubricas (pós-coordenação), como severidade (para dor, por exemplo) e temporalidade (agudo, subagudo e crônico), além de extensões regionais para detalhar rubricas com especificidades locais.

Desta forma, a CIAP-3 permitirá o registro das funcionalidades e o detalhamento de algumas condições. A regra da frequência foi manti$\mathrm{da}$, embora, para novas rubricas, como as de fun- cionalidade, a carência de dados as faz entrar por uma questão conceitual e epistemológica, o que já ocorreu com algumas da CIAP-2 (as rubricas -27 de cada capítulo são "medo de doença" e as rubricas -28 são "limitação funcional/ incapacidade").

Uma grande polêmica, que sempre acompanha as discussões de classificação em atenção primária, é como incluir "fatores de risco", uma vez que uma boa parte deles são "condições" por si só. Ou seja, enquanto alguns advogam pela criação de capítulos específicos para "fatores de risco", a decisão até aqui tem sido deixá-los nos respectivos capítulos como condições que podem ou não serem classificadas como fatores de risco nos diversos prontuários.

\section{Perspectivas futuras}

O mundo das classificações acabou se fundindo com o da informação, da inteligência artificial e do "bid data". A CID começou há pouco mais de 100 anos, como uma pequena lista de causas de morte. A CIAP tinha a intenção de ser apenas uma classificação de motivos de consulta. Ambas se desenvolveram muito no final do século XX. Hoje há uma disputa pelo controle da informação, sendo a estruturação dos dados parte importante deste processo. Estados Unidos da América, Inglaterra, Canadá e Austrália estão apostando no SNOMED International, que tem conseguido a adesão de cada vez mais países. Porém, por se tratar de uma complexa nomenclatura com mais de um milhão de termos, seu uso para estruturar os dados e devolvê-los como informação aos profissionais demanda sistemas de classificação que respeitem o campo de cada área, além de uma sofisticada informatização do processo de cuidado.

Por outro lado, a CID 11 foi lançada, mas ainda não implementada, o que está para ocorrer nos próximos anos. Seu sucesso é crucial para o posicionamento da OMS neste cenário de controle da informação e da epistemologia. Afinal, as classificações não devem ser usadas como protocolos, mas se as doenças e as condições não estão listadas, não existem estatisticamente. Neste sentido, a OMS, assim como fez com a parte de saúde mental da CID $10^{40}$, tem apontado na direção de organizar um "subset", ou seja, um recorte da CID 11 para uso específico na atenção primária. Porém, a experiência com a CID 10 não foi bem sucedida, pois cada capítulo das últimas versões da CID foram organizadas por especialistas focais, enquanto que a medicina geral, ou mesmo a 
atenção primária, não saíram fortalecidas ou bem representadas por grupos de especialistas. Assim, a OMS corre o risco de não valorizar a CIAP, que faz parte da sua própria "Família de Classificações" ${ }^{41}$, além de passar a mensagem de apoio a uma atenção primária fragmentada, formada por uma coleção de condições pouco frequentes ou sem uma base epistemológica consistente e, mais importante, não reconhecida como legítima por quem pratica e está na linha de frente.

\section{Conclusão}

Não há como prever como vão interagir as novas tecnologias, classificações, governos e organismos internacionais na disputa pelo controle da informação. Os profissionais que atuam na ponta precisam receber de volta os dados que levantaram, em forma de relatórios, informações úteis para a gestão da clínica. Só assim poderão se interessar pelo caminho que o dado faz desde que é comunicado pelo paciente até ser usado para predizer o risco de um próximo paciente. $\mathrm{E}$, então, profissionais da ponta e pacientes, juntos, poderão assumir o controle pela organização das informações e estas serem utilizadas de fato e exclusivamente para o benefício das pessoas. 


\section{Referências}

1. Classification Committee. World Organization of National Colleges, Academies and Academic Associations of General Practitioners/ Family Physicians (WONCA). International Classification of Health Problemas in Primary Care (ICHPPC-2-Defined). $3^{\text {th }}$ ed. Oxford: Oxford University Press; 1983.

2. White K. Introduction 3. In: World Organization of National Colleges Academies (WONCA). Academic Associations of General Practitioners. Family. Oxford: Oxford University Press; 1979.

3. Crombie DL. Diagnostic Process. J Coll Gen Practit 1963; 6:579-589.

4. Research Committee of the College of General Practitioners. A classification of disease. J R Coll Gen Pract 1959; 2:140-159.

5. Cypress BK. Patients' Reasons for Visiting Physicians: National Ambulatory Medical Care Survey. Washington: United States; 1977-1978. [Data the National Health Survey Series 13, No. 56].

6. Tenney J, White K, Williamson J. National Center for Health Statistics: National Ambulatory Medical Care Survey, background and methodology, United States, 1967-72. Vital and Health Statistics. Series 2-No. 61. DHEW Pub. No. (HRA) 74-1335. Health Resources Administration. Washington: U.S. Government Printing Office; 1974.

7. Meads S, McLemore T. National Center for Health Statistics: National Ambulatory Medical Care Survey: Symptom classification. Vital and Health Statistics. Series 2-No. 63. DHEW Pub. No. (HRA) 74-1337. Health Resources Administration. Washington: U.S. Government Printing Office; 1974.

8. Bentsen BG. Illness and general practice. A survey of medical care in an island population in South-East Norway. Oslo: Oslo University Press; 1970

9. Bridges-Webb C. Classification of disease in general practice. In: International Workshop on General Practice Research, Melbourne, 1972.

10. Westbury RC. A plan to develop an international classification of disease in family medicine. In: International Workshop on General Practice Research, Melbourne, 1972.

11. Sive P, Spencer T. Classification for cooperative morbidity survey, Unpublished, 1972.

12. Classification Committee of World Organization of National Colleges, Academies and Academic Associations of General Practitioners/ Family Physicians (WONCA) in collaboration with Classification Committee of North American Primary Care Research Group (NAPCRG). International Classification of Process in Primary Care (IC-Process-PC). Oxford: Oxford University Press; 1986.

13. The WONCA International Classification Committee. World Organization of National Colleges, Academies and Academic Associations of General Practitioners/ Family Physicians (WONCA). International Classification of Primary Care (ICPC-2-R). $2^{\text {nd }}$ ed. Revised. Oxford: Oxford University Press; 1998.

14. Lamberts H, Meads S, Wood M. Classification of reasons why persons seek primary care: pilot study of a new system. Public Health Report 1984; 99:597-605.
15. Meads S. The WHO Motivos da consulta Classification. Who Chronicle 1983; 37(5):159-162.

16. Lebrão ML. Classificação internacional de motivos de consulta para assistência primária: testes em algumas áreas brasileiras. Rev Saude Publica 1985; 19(1):69-78.

17. Lamberts H, Woods $M$. The birth of the International Classification of Primary Care (ICPC). Serendipity at the border of Lac Léman. Family Practice 2002; 19(5):433-435.

18. Bowker GC, Star SL. Sorting Things Out: Classification and Its Consequences. Cambridge: MIT Press; 1999.

19. Organização Mundial da Saúde (OMS). Como usar a CID. In. Organização Mundial da Saúde (OMS). CID10. Classificação Estatística Internacional de Doenças e Problemas Relacionados à Saúde. Décima Revisão. Volume 2: Manual de Instrução. São Paulo: Editora da Universidade de São Paulo, 2008. p. 21-34.

20. Meads S, McLemore T. The National Ambulatory Medical Care Survey: symptom classification. Rockville: Health Resources Administration; 1974. [DHEW publication No. (HRA) 74-1337].

21. Schneider D, Appleton L, McLemore T. A reason for visit classification for ambulatory care. Hyattsville: National Center for Health Statistics; 1978. [Vital and Health Statistics, Series 2, No. 78. DHEW publication No. (PHS)78-1352].

22. Schneider D. An ambulatory classification system: design development and evaluation. Health Serv. Res. 1979; 14(1):77-78.

23. Schneider D, Appleton L, McLemore T. A reason for visit classification for ambulatory care. Vital Health Statist 2 1979; (78):i-vi.

24. World Health Organization (WHO). International classification of procedures in medicine. Geneva: WHO; 1978.

25. North American Primary Care Research Group (NAPCRG). A process code for primary care (NAP$C R G-1)$. International field trial version. Richmond: NAPCRG; 1981.

26. Tindall HL, Culpepper L, Froom J, Henderson RA, Richards AD, Rosser WW, Wiegert HT. The NAPCRG process classification for primary care. J Fam Pract 1981; 12(2):309-318.

27. World Health Organization (WHO). Psychological factors affecting health assessment, classification and utilization workshop. Geneva: WHO; 1980. [Report of the World Health Organization on the conference at Bellagio, Italy, Nov. 6-10, 1979. Paper No. MNH 80].

28. Kupka K. International Classification of Diseases, ninth revision. WHO Chron 1978; 32:219-225.

29. Lamberts H, Wood M, editors. International Classification of Primary Care (ICPC). Oxford: Oxford University Press; 1987.

30. Lamberts H, Wood M, Hofmans-Okkes IM, editors. The International Classification of Primary Care in the European Community: with Multi-Language Layer. Oxford: Oxford University Press; 1993.

31. Sociedade Brasileira de Medicina de Família e Comunidade (SBMFC). CIAP 2. [homepage na Internet] [acessado 2019 Out 17]. Disponível em: https://www. sbmfc.org.br/ciap-2/ 
32. SNOMED International [homepage na Internet] [acessado 2019 Out 17]. Disponível em: http://www. snomed.org/

33. Tudorache T, Falconer SM, Nyulas CI, Noy NF, Musen MA. Will Semantic Web technologies work for the development of ICD-11? In: Ninth International Semantic Web Conference, Shanghai, China; 2010.

34. Weed LL. Medical records that guide and teach. $N$ Engl J Med 1968; 278:593-600, 652-657.

35. Soler JK, Okkes I. Reasons for encounter and symptom diagnoses: a superior description of patients' problems in contrast to medically unexplained symptoms (MUS). Fam Pract 2012; 29(3):272-282.

36. Gusso GDF, Benseñor IM. A methodological proposal to research patients' demands and pre-test probabilities using paper forms in primary care settings. Rev Bras Med Fam Comunidade. 2013; 8(27):97-105. Disponível em: http://dx.doi.org/10.5712/rbmfc8(27)692

37. Gusso GDF, Lotufo P, Benseñor IM. Assessment of pre-test probability in Primary Health Care using the International Classification of Primary Care - 2 (ICPC-2). Rev Bras Med Fam Comunidade 2013; 8(27):112-120.

38. World Organization of Family Doctors (WONCA) [homepage na Internet]. [acessado 2019 Out 17]. Disponível em: https://www.globalfamilydoctor.com/ News/January2019reportofWICC.aspx

39. Organização Mundial de Saúde (OMS). CIF: Classificação Internacional de Funcionalidade, Incapacidade e Saúde. São Paulo: EDUSP; 2003.

40. Organização Mundial da Saúde (OMS). CID 10- Diretrizes diagnósticas e de tratamento para transtornos mentais em cuidados primários. Porto Alegre: Artmed; 1998.

41. World Health Organization (WHO). [homepage na Internet]. [acessado 2019 Out 17]. Disponível em: https://www.who.int/classifications/en/

Artigo apresentado em 25/08/2019

Aprovado em 29/10/2019

Versão final apresentada em 31/10/2019 